LA-UR-93-474

hep-th/9302046

\title{
Constrained Dynamics of the Coupled Abelian Two-Form
}

\author{
Amitabha Lahiri ${ }^{\dagger}$ \\ Theoretical Division T-8 \\ Los Alamos National Laboratory \\ Los Alamos, NM 87545, USA
}

\begin{abstract}
I present the reduction of phase space of the theory of an antisymmetric tensor potential coupled to an abelian gauge field, using Dirac's procedure. Duality transformations on the reduced phase space are also discussed.
\end{abstract}

01/93

† (lahiri@pion.lanl.gov) 


\section{Introduction}

The uses of an abelian antisymmetric tensor potential is manifold. It was probably first used in the context of particle theory to describe a massless particle of zero helicity, the notoph [1,2]. It has subsequently appeared in the context of the theory of fundamental strings [3,4] and of cosmic strings and vortices [5, 6,67.7]. It has also been used to put a topological charge (hair) on black holes [8,9, 10], and in a mechanism for generating masses for vector bosons [11,12] via a derivative coupling. The use of the word 'abelian' in the description means that the antisymmetric potential $B_{\mu \nu}$ is invariant under gauge transformations belonging to any compact gauge group, although it does have an internal non-compact symmetry. (Nonabelian generalizations will not be discussed here.) It is also known that the free $B_{\mu \nu}$ field is dynamically 'dual' to a scalar, quantum mechanically [13,14] as well as classically.

In this letter, I apply Dirac's procedure [15] for reduction of phase space to the theory of an abelian antisymmetric tensor potential coupled to an abelian gauge field. The analysis has a couple of important differences with the corresponding analysis for the free $B_{\mu \nu}$, which has been known for a long time [16]. One important point is that in the coupled theory the gauge field $A_{\mu}$ provides a current for $B_{\mu \nu}$, and vice versa. As a result, choosing a gauge can be tricky. Another difference from the free theory (and an intriguing feature by itself) is that a duality transformation in the reduced phase space leads to very unusual Poisson brackets between the transformed fields and momenta.

The theory under consideration is given by the action

$$
S=\int d^{4} x\left(-\frac{1}{6} H_{\mu \nu \lambda} H^{\mu \nu \lambda}-\frac{1}{4} F_{\mu \nu} F^{\mu \nu}+\frac{m}{2} \epsilon^{\mu \nu \lambda \rho} B_{\mu \nu} F_{\lambda \rho}\right),
$$

where $F_{\mu \nu}=\partial_{[\mu} A_{\nu}=\partial_{\mu} A_{\nu}-\partial_{\nu} A_{\mu}$ and $H_{\mu \nu \lambda}=\partial_{\mu} B_{\nu \lambda}+\partial_{\nu} B_{\lambda \mu}+\partial_{\lambda} B_{\mu \nu}$. My convention in this paper will be $\epsilon^{0 i j k}=+\epsilon^{i j k} \equiv+\epsilon_{i j k}$, the metric is flat, $g_{\mu \nu}=\operatorname{diag}(-+++)$. (Thus $\left.\epsilon_{0 i j k}=-\epsilon_{i j k}.\right)$ Then the last term in the Lagrangian may be written as $m \epsilon_{i j k}\left(B_{0 i} F_{j k}+\right.$ $\left.B_{i j} F_{0 k}\right)$. This action remains invariant under the independent gauge transformations

$$
A_{\mu} \rightarrow A_{\mu}+\partial_{\mu} \chi, \quad B_{\mu \nu} \rightarrow B_{\mu \nu},
$$

and

$$
A_{\mu} \rightarrow A_{\mu}, \quad B_{\mu \nu} \rightarrow B_{\mu \nu}+\partial_{[\mu} \Lambda_{\nu]} .
$$

The Euler-Lagrange equations for $A_{\mu}$ and $B_{\mu \nu}$ are readily derived,

$$
\frac{\delta S}{\delta A_{\mu}}=\partial_{\nu} F^{\mu \nu}-\frac{m}{3} \epsilon^{\mu \nu \lambda \rho} H_{\nu \lambda \rho}=0
$$


and

$$
\frac{\delta S}{\delta B_{\mu \nu}}=\partial_{\lambda} H^{\mu \nu \lambda}+\frac{m}{2} \epsilon^{\mu \nu \lambda \rho} F_{\lambda \rho}=0 .
$$

Here my convention is $\delta B_{\mu \nu} / \delta B_{\rho \lambda}=\frac{1}{2}\left(g_{\mu \rho} g_{\nu \lambda}-g_{\mu \lambda} g_{\nu \rho}\right)$. Contracting (1.3) with $\epsilon_{\mu \nu \lambda \rho}$, substituting the resulting expression for $H_{\mu \nu \lambda}$ into (1.4) and using the Jacobi identity $\partial_{[\mu} F_{\nu \lambda]}=0$, one finally arrives at the wave equation

$$
\left(\square-2 m^{2}\right) F_{\mu \nu}=0 \text {. }
$$

This is the equation for a free massive object of mass $\sqrt{2} m$. As an aside, the action discussed in 11] has a factor of $-\frac{1}{12}$ instead of the $-\frac{1}{6}$ and $\frac{m}{4}$ instead of $\frac{m}{2}$. That produces a mass $m$ for the gauge field. The choice of factors in (1.1) is an arbitrary aesthetic choice, related to the choice of the factor of $\frac{1}{2}$ in the definition of $\delta B_{\mu \nu} / \delta B_{\rho \lambda}$. One may regain the so-called [14] 'standard' normalization by replacing $B_{\mu \nu}$ by $B_{\mu \nu} / \sqrt{2}, m$ by $m / \sqrt{2}$, and by removing the $\frac{1}{2}$ in the definition of $\delta B_{\mu \nu} / \delta B_{\rho \lambda}$.

\section{Constraints of the Theory}

The momenta conjugate to $A_{i}$ and $B_{i j}$ are respectively

$$
\Pi_{i}=\frac{\delta \mathcal{L}}{\delta \dot{A}_{i}}=F_{0 i}+m \epsilon_{i j k} B_{j k} ; \quad \Pi_{i j}=\frac{\delta \mathcal{L}}{\delta \dot{B}_{i j}}=H_{0 i j}
$$

The canonical Hamiltonian is then

$$
\begin{aligned}
\mathcal{H}_{C}= & \dot{A}_{i} \Pi_{i}+\dot{B}_{i j} \Pi_{i j}-\mathcal{L} \\
= & \Pi_{i} \Pi_{i}-m \epsilon_{i j k} B_{j k} \Pi_{i}+\partial_{i} A_{0} \Pi_{i}+\Pi_{i j} \Pi_{i j}-\left(\partial_{j} B_{0 i}-\partial_{i} B_{0 j}\right) \Pi_{i j}-\mathcal{L} \\
= & \frac{1}{2} \Pi_{i} \Pi_{i}+\frac{1}{2} \Pi_{i j} \Pi_{i j}+\frac{1}{6} H_{i j k} H_{i j k}+m^{2} B_{i j} B_{i j}+\frac{1}{4} F_{i j} F_{i j}-m \epsilon_{i j k} \Pi_{i} B_{j k} \\
& \quad+\partial_{i} A_{0} \Pi_{i}-\left(\partial_{j} B_{0 i}-\partial_{i} B_{0 j}\right) \Pi_{i j}-m \epsilon_{i j k} B_{0 i} F_{j k} .
\end{aligned}
$$

The primary constraints of this system are

$$
\Pi_{0} \approx 0, \quad \Pi_{0 i} \approx 0
$$

So I have to add Lagrange multiples of these constraints to the canonical Hamiltonian and integrate over all space to get a Hamiltonian

$$
H_{0}=\int d^{3} x\left(\mathcal{H}_{C}+v(\mathbf{x}) \Pi_{0}(\mathbf{x})+v_{i}(\mathbf{x}) \Pi_{0 i}(\mathbf{x})\right) .
$$


The canonical Poisson brackets are

$$
\begin{aligned}
\left\{A_{\mu}(\mathbf{x}), \Pi_{\nu}(\mathbf{y})\right\} & =g_{\mu \nu} \delta^{3}(\mathbf{x}-\mathbf{y}), \\
\left\{B_{\mu \nu}(\mathbf{x}), \Pi_{\rho \lambda}(\mathbf{y})\right\} & =\frac{1}{2}\left(g_{\mu \rho} g_{\nu \lambda}-g_{\mu \lambda} g_{\nu \rho}\right) \delta^{3}(\mathbf{x}-\mathbf{y}),
\end{aligned}
$$

all the other brackets being zero. Here and everywhere else, it is understood that brackets are taken at equal times. In addition, I will not specify the spatial dependence where it is obvious. Then the compatibility conditions for the primary constraints (2.3) are given by their Poisson brackets with the Hamiltonian $H_{0}$,

$$
\begin{aligned}
\dot{\Pi}_{0}=\left\{\Pi_{0}, H_{0}\right\} & =-\partial_{i} \Pi_{i} \approx 0 ; \\
\dot{\Pi}_{0 i}=\left\{\Pi_{0 i}, H_{0}\right\} & =\partial_{j} \Pi_{i j}-\frac{m}{2} \epsilon_{i j k} F_{j k} \approx 0 .
\end{aligned}
$$

As is obvious, these are respectively the 0 -th and [0i]-th components of the Euler-Lagrange equations. These are the secondary constraints of the system, and one can read off that the Poisson brackets of (2.6) with (2.3) vanish. Therefore these are all first-class constraints.

Now Lagrange multiples of these secondary constraints are added to $H_{0}$ to get the unconstrained Hamiltonian

$$
H=H_{0}+\int d^{3} x\left(w(\mathbf{x}) \partial_{i} \Pi_{i}+w_{i}(\mathbf{x})\left(\partial_{j} \Pi_{i j}-\frac{m}{2} \epsilon_{i j k} F_{j k}\right)\right) .
$$

The Lagrange multipliers $v, v_{i}, w$ and $w_{i}$ may be computed from the Poisson brackets of the fields with this Hamiltonian,

$$
\begin{aligned}
\dot{A}_{0} & =\left\{A_{0}, H\right\}=-v \\
\dot{A}_{i} & =\left\{A_{i}, H\right\}=\Pi_{i}+\partial_{i} A_{0}-\partial_{i} w-m \epsilon_{i j k} B_{j k} \\
\dot{B}_{0 i} & =\left\{B_{0 i}, H\right\}=-\frac{1}{2} v_{i} \\
\dot{B}_{i j} & =\left\{B_{i j}, H\right\}=\Pi_{i j}-\partial_{j} B_{0 i}+\partial_{i} B_{0 j}-\frac{1}{2}\left(\partial_{j} w_{i}-\partial_{i} w_{j}\right) .
\end{aligned}
$$

Therefore one may set $v=-\dot{A}_{0}, v_{i}=-2 \dot{B}_{0 i}, w=0$, and $w_{i}=0$. (Actually, instead of $w=0$ and $w_{i}=0$ it is sufficient to set $\partial_{i} w=0$ and $\partial_{[i} w_{j]}=0$. The resulting Hamiltonian then differs from the one below by boundary terms corresponding to the 'zero modes' of the system. I will neglect these boundary terms in what follows. (For an account of how the boundary terms affect the vacuum structure of the quantum theory, and related topological issues, see [17,18].) Finally, one may compute the Poisson bracket of the secondary constraints with the Hamiltonian, $\left\{\partial_{i} \Pi_{i}, H\right\} \approx \partial_{i} \partial_{j} F_{i j}=0$, while

$$
\begin{gathered}
\left\{\partial_{i} \Pi_{i j}-\frac{m}{2} \epsilon_{i j k} F_{j k}, H\right\} \approx 2 m^{2} \partial_{j} B_{k l} \frac{1}{2}\left(\delta_{i k} \delta_{j l}-\delta_{i l} \delta_{j k}\right)-m \epsilon_{k l m} \partial_{j} \Pi_{k} \frac{1}{2}\left(\delta_{i l} \delta_{j m}-\delta_{i m} \delta_{j l}\right) \\
+m \epsilon_{i j k} \partial_{j} \Pi_{k}-m^{2} \epsilon_{i j k} \epsilon_{k l m} \partial_{j} B_{l m}=0 .
\end{gathered}
$$

Therefore there are no tertiary constraints, and (2.3) and (2.6) form a complete set of first-class constraints. 


\section{Constrained Hamiltonian}

In order to compute Dirac brackets for the system, I shall fix the gauge by choosing 'gauge-fixing' constraints with non-vanishing Poisson brackets with the primary and secondary constraints (2.3) and (2.6). The 0-th component of the Euler-Lagrange equations for (1.3) reads

$$
-\partial_{0} \partial_{i} A_{i}+\nabla^{2} A_{0}-\frac{m}{3} \epsilon_{i j k} H_{i j k}=0 .
$$

It follows from this equation that the gauge fixing constraints $A_{0} \approx 0$ and $\partial_{i} A_{i} \approx 0$ cannot both be imposed (unless $m=0$ ). I will choose 'radiation gauge' constraints

$$
\partial_{i} A_{i} \approx 0 \quad \text { and } \quad A_{0}(\mathbf{x})+\frac{m}{3} \int \frac{d^{3} y}{4 \pi|\mathbf{x}-\mathbf{y}|} \epsilon_{i j k} H_{i j k}(\mathbf{y}) \approx 0 .
$$

This gauge-fixing can be done by performing the following gauge transformations, first

$$
A_{\mu} \rightarrow A_{\mu}^{\prime}(\mathbf{x})=A_{\mu}(\mathbf{x})-\partial_{\mu} \int_{0}^{x_{0}} d t\left[A_{0}(\mathbf{x}, t)+\frac{m}{3} \int \frac{d^{3} y}{4 \pi|\mathbf{x}-\mathbf{y}|} \epsilon_{i j k} H_{i j k}(\mathbf{y}, t)\right],
$$

so that

$$
A_{0}^{\prime}+\frac{m}{3} \int \frac{d^{3} y}{4 \pi|\mathbf{x}-\mathbf{y}|} \epsilon_{i j k} H_{i j k}(\mathbf{y})=0,
$$

and (3.1) becomes $\partial_{0} \partial_{i} A_{i}^{\prime}=0$, where I have used $\nabla_{x}^{2}\left(\frac{1}{4 \pi|\mathbf{x}-\mathbf{y}|}\right)=-\delta^{3}(\mathbf{x}-\mathbf{y})$. Then another gauge transformation is made,

$$
A_{\mu}^{\prime} \rightarrow A_{\mu}^{\prime \prime}(\mathbf{x})=A_{\mu}^{\prime}(\mathbf{x})+\partial_{\mu} \int \frac{d^{3} y}{4 \pi|\mathbf{x}-\mathbf{y}|} \partial_{i}^{y} A_{i}^{\prime}(\mathbf{y})
$$

Then $\partial_{i} A_{i}^{\prime \prime}=0$ and $A_{0}^{\prime \prime}=A_{0}^{\prime}$, where I have used $\partial_{0} \partial_{i} A_{i}^{\prime}=0$.

Similarly, the $0 i$-th component of the Euler-Lagrange equations (1.4) reads

$$
-\partial_{0} \partial_{j} B_{i j}-\nabla^{2} B_{0 i}+\partial_{i} \partial_{j} B_{0 j}+\frac{m}{2} \epsilon_{i j k} F_{j k}=0 .
$$

Again, $B_{0 i} \approx 0$ and $\partial_{j} B_{i j} \approx 0$ cannot both be imposed unless $m=0$. I shall choose what may be called 'radiation gauge' constraints for the $B_{\mu \nu}$ field,

$$
\partial_{j} B_{i j} \approx 0 \quad \text { and } \quad B_{0 i}+\frac{m}{2} \int \frac{d^{3} y}{4 \pi|\mathbf{x}-\mathbf{y}|} \epsilon_{i j k} F_{j k}(\mathbf{y}) \approx 0 .
$$

These choices can be made via the following gauge transformations (see (1.2)), first

$$
B_{\mu \nu} \rightarrow B_{\mu \nu}^{\prime}(\mathbf{x})=B_{\mu \nu}(\mathbf{x})+\partial_{[\mu} \int_{0}^{x_{0}} d t\left[B_{\nu] 0}(\mathbf{x}, t)-\frac{m}{2} \int \frac{d^{3} y}{4 \pi|\mathbf{x}-\mathbf{y}|} \epsilon_{\nu] 0 j k} F_{j k}(\mathbf{y}, t)\right]
$$


so that

$$
B_{0 i}^{\prime}+\frac{m}{2} \int \frac{d^{3} y}{4 \pi|\mathbf{x}-\mathbf{y}|} \epsilon_{i j k} F_{j k}(\mathbf{y})=0,
$$

and (3.6) is now $\partial_{0} \partial_{j} B_{i j}^{\prime}=0$. Then the radiation gauge is achieved via another gauge transformation

$$
B_{\mu \nu}^{\prime} \rightarrow B_{\mu \nu}^{\prime \prime}=B_{\mu \nu}^{\prime}-\partial_{\mu} \int \frac{d^{3} y}{4 \pi|\mathbf{x}-\mathbf{y}|} \partial_{k}^{y} B_{\nu k}^{\prime}+\partial_{\nu} \int \frac{d^{3} y}{4 \pi|\mathbf{x}-\mathbf{y}|} \partial_{k}^{y} B_{\mu k}^{\prime}
$$

In this gauge, $\partial_{j} B_{i j}^{\prime \prime}=0$ and $B_{0 i}^{\prime \prime}=B_{0 i}^{\prime}$.

Now I can drop all primes and write down the full set of second-class constraints,

$$
\begin{aligned}
\phi_{1} & \equiv A_{0}(\mathbf{x})+\frac{m}{3} \int \frac{d^{3} y}{4 \pi|\mathbf{x}-\mathbf{y}|} \epsilon_{i j k} H_{i j k}(\mathbf{y}) \approx 0 \\
\phi_{2} & \equiv \Pi_{0} \approx 0 \\
\phi_{3} & \equiv \partial_{i} A_{i} \approx 0 \\
\phi_{4} & \equiv \partial_{i} \Pi_{i} \approx 0 \\
\phi_{5 i} & \equiv B_{0 i}+\frac{m}{2} \int \frac{d^{3} y}{4 \pi|\mathbf{x}-\mathbf{y}|} \epsilon_{i j k} F_{j k}(\mathbf{y}) \approx 0 \\
\phi_{6 i} & \equiv \Pi_{0 i} \approx 0, \\
\phi_{7 i} & \equiv \partial_{j} B_{i j} \approx 0 \\
\phi_{8 i} & \equiv \partial_{j} \Pi_{i j}-\frac{m}{2} \epsilon_{i j k} F_{j k} \approx 0 .
\end{aligned}
$$

The matrix of Poisson brackets of these constraints are easily computed from the canonical Poisson brackets (2.5). In particular, the matrix of Poisson brackets of the constraints $\phi_{7 i}$ and $\phi_{8 i}$ is

$$
\begin{aligned}
\left\{\phi_{7 i}(\mathbf{x}), \phi_{8 j}(\mathbf{y})\right\} & =\partial_{k}^{x} \partial_{l}^{y} \frac{1}{2}\left(g_{i j} g_{k l}-g_{i l} g_{j k}\right) \delta^{3}(\mathbf{x}-\mathbf{y}) \\
& =-\frac{1}{2}\left(\nabla_{x}^{2} g_{i j}-\partial_{i}^{x} \partial_{j}^{x}\right) \delta^{3}(\mathbf{x}-\mathbf{y})
\end{aligned}
$$

As is well-known, the right-hand side of this equation is non-invertible. It follows that the constraints $\phi_{7 i}$ and $\phi_{8 i}$ are degenerate. This degeneracy is due to the fact that there is a propagating mode of the $B_{\mu \nu}$ field. In four dimensions, the antisymmetric $B_{\mu \nu}$ has six independent components. On the other hand it appears as if there are six first-class constraints (2.3) and (2.6) on these components. These, however, are not independent constraints and their interdependence is carried through after gauge fixing into the second class constraints (3.11). The non-invertibility of the matrix of Poisson brackets shows that (3.11) is overcounting the number of second-class constraints. In order to compute the 
Dirac brackets of the fields, one needs the inverse of the matrix of the Poisson brackets of the second-class constraints. Because of the non-invertibility of (3.12) one has to resort to a trick. This trick is to introduce a parameter $\eta$, and redefine the relevant Poisson brackets,

$$
\left\{B_{\mu \nu}(\mathbf{x}), \Pi_{\rho \lambda}(\mathbf{y})\right\}=\frac{1}{2}\left(g_{\mu \rho} g_{\nu \lambda}-\left(1-\frac{1}{\eta}\right) g_{\mu \lambda} g_{\nu \rho}\right) \delta^{3}(\mathbf{x}-\mathbf{y}) .
$$

Then the Poisson brackets of $\phi_{7 i}$ and $\phi_{8 i}$ are given by

$$
\left\{\phi_{7 i}(\mathbf{x}), \phi_{8 j}(\mathbf{y})\right\}=-\frac{1}{2}\left(\nabla_{x}^{2} g_{i j}-\left(1-\frac{1}{\eta}\right) \partial_{i}^{x} \partial_{j}^{x}\right) \delta^{3}(\mathbf{x}-\mathbf{y})=:-\frac{1}{2} G_{i j}(\mathbf{x}) \delta^{3}(\mathbf{x}-\mathbf{y}) .
$$

I will compute the Dirac brackets as usual, then take the limit $\eta \rightarrow \infty$ at the end. The matrix of Poisson brackets of the constraints (3.11) is now given by

$$
\mathcal{C}_{\alpha \beta}(\mathbf{z}, \mathbf{w})=\left(\begin{array}{cccccccc}
0 & -1 & 0 & 0 & 0 & 0 & 0 & 0 \\
1 & 0 & 0 & 0 & 0 & 0 & 0 & 0 \\
0 & 0 & 0 & -\nabla_{z}^{2} & 0 & 0 & 0 & 0 \\
0 & 0 & \nabla_{z}^{2} & 0 & 0 & 0 & 0 & 0 \\
0 & 0 & 0 & 0 & 0 & -\frac{1}{2} g_{i j} & 0 & 0 \\
0 & 0 & 0 & 0 & \frac{1}{2} g_{i j} & 0 & 0 & 0 \\
0 & 0 & 0 & 0 & 0 & 0 & 0 & -\frac{1}{2} G_{i j}(\mathbf{z}) \\
0 & 0 & 0 & 0 & 0 & 0 & \frac{1}{2} G_{i j}(\mathbf{z}) & 0
\end{array}\right) \delta^{3}(\mathbf{z}-\mathbf{w}),
$$

where $\alpha, \beta=1, \cdots, 8$. The inverse of this matrix is easily computed to be

$$
\mathcal{C}_{\alpha \beta}^{-1}(\mathbf{z}, \mathbf{w})=\left(\begin{array}{cccccccc}
0 & 1 & 0 & 0 & 0 & 0 & 0 & 0 \\
-1 & 0 & 0 & 0 & 0 & 0 & 0 & 0 \\
0 & 0 & 0 & \frac{1}{\nabla_{z}^{2}} & 0 & 0 & 0 & 0 \\
0 & 0 & -\frac{1}{\nabla_{z}^{2}} & 0 & 0 & 0 & 0 & 0 \\
0 & 0 & 0 & 0 & 0 & 2 g_{i j} & 0 & 0 \\
0 & 0 & 0 & 0 & -2 g_{i j} & 0 & 0 & 0 \\
0 & 0 & 0 & 0 & 0 & 0 & 0 & 2 G_{i j}^{-1}(\mathbf{z}) \\
0 & 0 & 0 & 0 & 0 & 0 & -2 G_{i j}^{-1}(\mathbf{z}) & 0
\end{array}\right) \delta^{3}(\mathbf{z}-\mathbf{w}),
$$

where $\frac{1}{\nabla_{z}^{2}} \delta^{3}(\mathbf{z}-\mathbf{w})=-\frac{1}{4 \pi|\mathbf{z}-\mathbf{w}|}$, and $G_{i j}^{-1}$ is a formal inverse,

$$
G_{i j}^{-1}(\mathbf{z})=\left(g_{i j}-(1-\eta) \frac{\partial_{i}^{z} \partial_{j}^{z}}{\nabla_{z}^{2}}\right) \frac{1}{\nabla_{z}^{2}} .
$$

According to Dirac's prescription, the brackets among two objects $\mathcal{A}$ and $\mathcal{B}$ may now be calculated, consistent with setting the constraints (3.11) strongly to zero, by the formula

$$
\{\mathcal{A}(\mathbf{x}), \mathcal{B}(\mathbf{y})\}_{D}=\{\mathcal{A}(\mathbf{x}), \mathcal{B}(\mathbf{y})\}-\int d^{3} z d^{3} w\left\{\mathcal{A}(\mathbf{x}), \phi_{\alpha}(\mathbf{z})\right\} \mathcal{C}_{\alpha \beta}^{-1}(\mathbf{z}-\mathbf{w})\left\{\phi_{\beta}(\mathbf{w}), \mathcal{B}(\mathbf{y})\right\},
$$


where the brackets on the right hand side of the equation are the canonical Poisson brackets given in (2.5). The Dirac brackets of $A_{\mu}$ and $\Pi_{\nu}$ can be easily computed,

$$
\left\{A_{\mu}(\mathbf{x}), \Pi_{\nu}(\mathbf{y})\right\}_{D}=\left(g_{\mu \nu}+g_{0 \mu} g_{0 \nu}\right) \delta^{3}(\mathbf{x}-\mathbf{y})-\partial_{\mu}^{x} \partial_{\nu}^{y} \frac{1}{4 \pi|\mathbf{x}-\mathbf{y}|}
$$

For the calculation of the Dirac brackets of $B_{\mu \nu}$ and $\Pi_{\rho \lambda}$, one calculates with $\mathcal{C}_{\alpha \beta}^{-1}$ as given in (3.16) and then takes $\eta \rightarrow \infty$. Thus

$$
\begin{aligned}
\left\{B_{\mu \nu}(\mathbf{x}), \Pi_{\rho \lambda}(\mathbf{y})\right\}_{D}^{\eta}= & \frac{1}{2}\left(g_{\mu \rho} g_{\nu \lambda}-g_{\mu \lambda} g_{\nu \rho}\right) \delta^{3}(\mathbf{x}-\mathbf{y}) \\
& +\frac{1}{2}\left[g_{\mu 0} g_{0 \rho} g_{i \nu} g_{i \lambda}-\left(1-\frac{1}{\eta}\right) g_{\nu 0} g_{0 \rho} g_{i \mu} g_{i \lambda}\right. \\
& \left.-\left(1-\frac{1}{\eta}\right) g_{\mu 0} g_{0 \lambda} g_{i \nu} g_{i \rho}+\left(1-\frac{1}{\eta}\right)^{2} g_{\nu 0} g_{0 \lambda} g_{i \mu} g_{i \rho}\right] \delta^{3}(\mathbf{x}-\mathbf{y}) \\
& -\frac{1}{2}\left[\left(g_{\mu \rho}+g_{\mu 0} g_{0 \rho}\right) \partial_{\nu}^{x} \partial_{\lambda}^{y}-\left(1-\frac{1}{\eta}\right)\left(g_{\mu \lambda}+g_{\mu 0} g_{0 \lambda}\right) \partial_{\nu}^{x} \partial_{\rho}^{y}\right. \\
& -\left(1-\frac{1}{\eta}\right)\left(g_{\nu \rho}+g_{\nu 0} g_{0 \rho}\right) \partial_{\mu}^{x} \partial_{\lambda}^{y} \\
& \left.+\left(1-\frac{1}{\eta}\right)^{2}\left(g_{\nu \lambda}+g_{\nu 0} g_{0 \lambda}\right) \partial_{\mu}^{x} \partial_{\rho}^{y}\right] \frac{1}{4 \pi|\mathbf{x}-\mathbf{y}|} \\
& +\frac{1}{\eta^{2}} \partial_{\mu}^{x} \partial_{\nu}^{x} \partial_{\rho}^{y} \partial_{\lambda}^{y}\left(\frac{1-\eta}{\nabla_{x}^{2}}\right) \frac{1}{4 \pi|\mathbf{x}-\mathbf{y}|},
\end{aligned}
$$

for which one takes $\eta \rightarrow \infty$ and obtains the Dirac brackets for $B_{\mu \nu}$ and $\Pi_{\rho \lambda}$,

$$
\begin{aligned}
\left\{B_{\mu \nu}(\mathbf{x}), \Pi_{\rho \lambda}(\mathbf{y})\right\}_{D}= & \frac{1}{2}\left(g_{\mu \rho} g_{\nu \lambda}-g_{\mu \lambda} g_{\nu \rho}\right) \delta^{3}(\mathbf{x}-\mathbf{y}) \\
& +\frac{1}{2}\left(g_{\mu 0} g_{0 \rho} g_{\nu \lambda}-g_{\nu 0} g_{0 \rho} g_{\mu \lambda}-g_{\mu 0} g_{0 \lambda} g_{\nu \rho}+g_{\nu 0} g_{0 \lambda} g_{\mu \rho}\right) \delta^{3}(\mathbf{x}-\mathbf{y}) \\
& -\frac{1}{2}\left[\left(g_{\mu \rho}+g_{\mu 0} g_{0 \rho}\right) \partial_{\nu}^{x} \partial_{\lambda}^{y}-\left(g_{\mu \lambda}+g_{\mu 0} g_{0 \lambda}\right) \partial_{\nu}^{x} \partial_{\rho}^{y}\right. \\
& \left.-\left(g_{\nu \rho}+g_{\nu 0} g_{0 \rho}\right) \partial_{\mu}^{x} \partial_{\lambda}^{y}+\left(g_{\nu \lambda}+g_{\nu 0} g_{0 \lambda}\right) \partial_{\mu}^{x} \partial_{\rho}^{y}\right] \frac{1}{4 \pi|\mathbf{x}-\mathbf{y}|} .
\end{aligned}
$$

The other Dirac brackets may be computed in a similar fashion, and they are

$$
\begin{gathered}
\left\{A_{\mu}(\mathbf{x}), \Pi_{\nu \lambda}(\mathbf{y})\right\}_{D}=2 m g_{0 \mu} \epsilon_{\nu \lambda \rho} \partial_{\rho}^{y} \frac{1}{4 \pi|\mathbf{x}-\mathbf{y}|} \\
\left\{B_{\mu \nu}(\mathbf{x}), \Pi_{\lambda}(\mathbf{y})\right\}_{D}=m\left(g_{0 \nu} \epsilon_{\lambda \mu \sigma}-g_{0 \mu} \epsilon_{\lambda \nu \sigma}\right) \partial_{\sigma}^{y} \frac{1}{4 \pi|\mathbf{x}-\mathbf{y}|} \\
\left\{\Pi_{\mu}(\mathbf{x}), \Pi_{\nu \lambda}(\mathbf{y})\right\}_{D}=m\left(\epsilon_{\mu \lambda \sigma} \partial_{\sigma}^{x} \partial_{\nu}^{y}-\epsilon_{\mu \nu \sigma} \partial_{\sigma}^{x} \partial_{\lambda}^{y}\right) \frac{1}{4 \pi|\mathbf{x}-\mathbf{y}|} \\
\left\{A_{\mu}, A_{\nu}\right\}_{D}=\left\{A_{\mu}, B_{\mu \nu}\right\}_{D}=\left\{B_{\mu \nu}, B_{\rho \lambda}\right\}_{D}=\left\{\Pi_{\mu}, \Pi_{\nu}\right\}_{D}=\left\{\Pi_{\mu}, \Pi_{\mu \nu}\right\}_{D}=0 .
\end{gathered}
$$


Here $\epsilon_{\mu \nu \lambda}=\epsilon_{i j k} g_{i \mu} g_{j \nu} g_{k \lambda}$.

One may then set the constraints (3.11) strongly to zero. The fully constrained Hamiltonian is then

$$
H=\int d^{3} x\left(\frac{1}{2} \Pi_{i} \Pi_{i}+\frac{1}{2} \Pi_{i j} \Pi_{i j}+\frac{1}{6} H_{i j k} H_{i j k}+\frac{1}{4} F_{i j} F_{i j}+m^{2} B_{i j} B_{i j}-m \epsilon_{i j k} \Pi_{i} B_{j k}\right),
$$

with the fields and the momenta obeying the Dirac brackets as given by $(3.19)-(3.22)$. The linear nature of the coupling between $\Pi_{i}$ and $B_{j k}$ may raise questions as to the boundedness (from below) of this Hamiltonian. However, (3.23) is bounded from below, since

$$
\begin{aligned}
H & \equiv \int d^{3} x\left(\frac{1}{2}\left(\Pi_{i}-m \epsilon_{i j k} B_{j k}\right)\left(\Pi_{i}-m \epsilon_{i l m} B_{l m}\right)+\frac{1}{2} \Pi_{i j} \Pi_{i j}+\frac{1}{6} H_{i j k} H_{i j k}+\frac{1}{4} F_{i j} F_{i j}\right) \\
& =\int d^{3} x\left(\frac{1}{2} F_{0 i} F_{0 i}+\frac{1}{2} \Pi_{i j} \Pi_{i j}+\frac{1}{6} H_{i j k} H_{i j k}+\frac{1}{4} F_{i j} F_{i j}\right)
\end{aligned}
$$

which is a sum of squares.

The duality between the antisymmetric potential and a scalar is quite interesting in this formalism. First, let $\Pi_{i}^{\prime}$ be defined as $\Pi_{i}^{\prime}:=F_{0 i}=\Pi_{i}-m \epsilon_{i j k} B_{j k}$ (a non-canonical transformation). Then from (3.21) and (3.22), one calculates that

$$
\left\{\Pi_{i}^{\prime}(\mathbf{x}), \Pi_{j k}(\mathbf{y})\right\}_{D}=-m \epsilon_{i j k} \delta^{3}(\mathbf{x}-\mathbf{y})
$$

On the other hand, $\Pi_{i}^{\prime}$ has the same Dirac brackets as $\Pi_{i}$ with all the other variables that appear in the Hamiltonian (3.23). I now drop the primes and rewrite the Hamiltonian as

$$
H=\int d^{3} x\left(\frac{1}{2} \Pi_{i} \Pi_{i}+\frac{1}{2} \Pi_{i j} \Pi_{i j}+\frac{1}{6} H_{i j k} H_{i j k}+\frac{1}{4} F_{i j} F_{i j}\right) .
$$

The fields are now uncoupled in the Hamiltonian but coupled through their Dirac brackets (3.25). Even though this was not a canonical transformation, no information has been lost. Now I make a canonical transformation from the variables $\left(B_{\mu \nu}, \Pi_{\rho \lambda}\right)$ to the scalar variables $\left(\phi, \Pi_{\phi}\right)$,

$$
\phi(\mathbf{x})=\frac{1}{\sqrt{2}} \epsilon_{i j k} \int_{x}^{\infty} d \lambda \frac{d z_{i}}{d \lambda} \Pi_{j k}(\mathbf{z}), \quad \Pi_{\phi}=\frac{1}{\sqrt{2}} \epsilon_{i j k} \partial_{i} B_{j k} .
$$

The inverse transformation is also non-local,

$$
\Pi_{i j}=\frac{1}{\sqrt{2}} \epsilon_{i j k} \partial_{k} \phi, \quad B_{i j}(\mathbf{x})=\frac{1}{3 \sqrt{2}} \epsilon_{i j k} \int_{x}^{\infty} d \lambda \frac{d z_{k}}{d \lambda} \Pi_{\phi}
$$


The hamiltonian may now be rewritten in terms of the variables $\left(A_{i}, \Pi_{i}, \phi, \Pi_{\phi}\right)$,

$$
H=\int d^{3} x\left(\frac{1}{2} \Pi_{i} \Pi_{i}+\frac{1}{4} F_{i j} F_{i j}+\frac{1}{2} \Pi_{\phi} \Pi_{\phi}+\frac{1}{2} \partial_{i} \phi \partial_{i} \phi\right),
$$

with Dirac brackets given by (3.19) (where $\Pi_{i}$ are the new $\Pi_{i}$ ), and

$$
\begin{aligned}
\left\{\phi(\mathbf{x}), \Pi_{\phi}(\mathbf{y})\right\}_{D} & =\delta^{3}(\mathbf{x}-\mathbf{y}) \\
\left\{A_{i}, \phi\right\}_{D}=\left\{A_{i}, \Pi_{\phi}\right\}_{D} & =\left\{\Pi_{i}, \Pi_{\phi}\right\}_{D}=0 \\
\left\{\Pi_{i}(\mathbf{x}), \phi(\mathbf{y})\right\}_{D} & =m \sqrt{2} \int_{y}^{\infty} d z_{i} \delta^{3}(\mathbf{x}-\mathbf{z}) .
\end{aligned}
$$

While $\left(B_{\mu \nu}, \Pi_{\rho \lambda}\right) \rightarrow\left(\phi, \Pi_{\phi}\right)$ is a canonical transformation, it is also non-local, and therefore incomplete. The systems (3.26) and (3.29) carry different topological information, as is evident from the brackets (3.30). While the brackets in the first system were local, the brackets now depend on a line of integration. Various authors [6, 4, 14, 17, 19] have pointed out relations between cosmic strings in the system (1.1) and local strings of the abelian Higgs model in the symmetry broken phase. The transformations (3.27), (3.28) and the Poisson brackets (3.30) seem to point to yet another description of the duality between the two systems. 


\section{References}

[1] V. I. Ogievetskii and I. V. Polubarinov, Sov. J. Nucl. Phys. (Iad. Fiz.) 4 (1967) 156.

[2] S. Deser, Phys. Rev. 187 (1969) 1931.

[3] M. Kalb and P. Ramond, Phys. Rev. D9 (1974) 2273.

[4] R. Rohm and E. Witten, Ann. Phys. 170 (1986) 454.

[5] A. Vilenkin and T. Vachaspati, Phys. Rev. D35 (1987) 1138.

[6] R. L. Davis and E. P. S. Shellard, Phys. Lett. B214 (1988) 219; Phys. Rev. Lett. 63 (1989) 2021.

[7] R. L. Davis, Phys. Rev. D40 (1989) 4033; Mod. Phys. Lett. A5 (1990) 955.

[8] M. J. Bowick et al., Phys. Rev. Lett. 61 (1988) 2823.

[9] T. J. Allen, M. J. Bowick and A. Lahiri, Phys. Lett. B237 (1989) 47.

[10] Bruce A. Campbell,et al., Phys. Lett. B251 (1990) 34; Nucl. Phys. B351 (1991) 778.

[11] T. J. Allen, M. J. Bowick and A. Lahiri, Mod. Phys. Lett. A6 (1991) 559.

[12] J. Minahan and R. Warner, Stuckelberg Revisited, University of Florida preprint UFIFT-HEP-89-15 (unpublished).

[13] C. P. Burgess and A. Kshirsagar, Nucl.Phys. B324 (1989) 157;

J. M. Molera and B. A. Ovrut, Phys. Rev. D40 (1989) 1146.

[14] K. Lee, "The Dual Formulation of Cosmic Strings and Vortices", Columbia U. preprint CU-TP-588, hep-th: 9301102.

[15] P. A. M. Dirac, Can. J. Math. 2 (1950) 129; Proc. Roy. Soc. A246 (1968) 326;

A. J. Hanson, T. Regge and C. Teitelboim, Constrained Hamiltonian Systems, Accad. Naz. dei Lincei, Rome, 1976.

[16] R. K. Kaul, Phys. Rev. D18 (1978) 1127.

[17] T. J. Allen, "Duality and the Vacuum", Nucl. Phys. B (to be published).

[18] A. P. Balachandran and P. Teotonio-Sobrinho, "The Edge states of the BF system and the London equations", Int. J. Mod. Phys. A (to be published).

[19] S. Coleman, J. Preskill and F. Wilczek, Nucl. Phys. B378 (1992) 175. 